\title{
Capacities in Generalized Orlicz Spaces
}

\author{
Debangana Baruah, ${ }^{1}$ Petteri Harjulehto, ${ }^{1}$ and Peter Hästö $\mathbb{D I D}^{1,2}$ \\ ${ }^{1}$ Department of Mathematics and Statistics, 20014, University of Turku, Finland \\ ${ }^{2}$ Department of Mathematics, 90014, University of Oulu, Finland \\ Correspondence should be addressed to Peter Hästö; peter.hasto@oulu.fi
}

Received 28 June 2018; Accepted 9 September 2018; Published 1 October 2018

Academic Editor: Alberto Fiorenza

Copyright (C) 2018 Debangana Baruah et al. This is an open access article distributed under the Creative Commons Attribution License, which permits unrestricted use, distribution, and reproduction in any medium, provided the original work is properly cited.

In this paper basic properties of both Sobolev and relative capacities are studied in generalized Orlicz spaces. The capacities are compared with each other and the Hausdorff measure. As an application, the existence of quasicontinuous representative of generalized Orlicz functions is proved.

\section{Introduction}

In the calculus of variations one studies existence and properties of solutions to minimization problems such as

$$
\min _{u \in W^{1,1}} \int F(x,|\nabla u|) d x
$$

Classical techniques, by De Giorgi and Moser, cover both the linear case and the $p$-growth case, where $F(t) \approx t^{p}$. Marcellini [1] developed the theory of $(p, q)$-growth, which is based on the growth assumption $t^{p}-1 \leqslant F(x, t) \leqslant t^{q}+1, q>p$. Zhikov [2] studied such minimizers as models of anisotropic materials and also observed that they exhibit the so-called Lavrentiev phenomenon whereby minimizers do not have improved regularity and may even be discontinuous.

In the variable exponent case, $F(x, t) \approx t^{p(x)}$, the change in the anisotropy (growth rate) is gradual owing to the continuity of $p$. For instance, in electrorheological fluid dynamics, where the anisotropy depends on the smooth electrical field, this is a reasonable assumption [3]. In other situations, such as composite materials, a more clear-cut transition is better. To this end, Baroni, Colombo, and Mingione [4-7] have developed a regularity theory of the double phase functional $F(x, t)=t^{p}+a(x) t^{q}, q>p$, which has the property that the growth rate changes abruptly from $p$ to $q$ in the sets $\{a=0\}$ and $\{a>0\}$ (see also [8-12]). Recently, we were able to generalize their first step, showing Hölder continuity, to the general $\Phi$-growth case [13]. Also other results have recently been obtained for partial differential equations with generalized Orlicz growth, cf. [13-16].

A different approach to differential equations is based on (nonlinear) potential theory. The foundation of nonlinear potential theory includes general notions of a Radon measure, a capacity and generalized functions. The sets of capacity zero are the exceptional sets for representatives of the function. In this paper we give basic properties of both Sobolev capacity and relative capacity in the generalized Orlicz setting. We follow the general framework of [17] and its previous adaption to the variable exponent setting [18, Chapter 10]. The results can be applied, e.g., in the study of boundary behavior of solutions to PDE.

We consider general $\Phi$-functions which need not be convex. Many of the proofs in this paper follow a standard pattern, since they do not depend on the exact form of the integrand used in the definition of capacity. We have therefore omitted or abbreviated several proofs (e.g., Section 6). Nevertheless, it is necessary to check these basic building blocks in order to proceed with constructing the theory, since the results are not covered by earlier results, merely similar. There are also some proofs which are new, namely, Theorem 9, Example 13, and Proposition 20. Furthermore, this general setting clarifies the necessity of various assumptions for different properties (e.g., Theorem 8). In particular, we find that the relative capacity is Choquet also for nonconvex $\Phi$-function, whereas convexity is needed for the Sobolev capacity. 
It should be noted that Ohno and Shimomura [19] have recently studied (Sobolev) capacity in the generalized Orlicz case. However, they consider the capacity in a metric measure space setting with Hajłasz gradients. These results therefore work in the Euclidean setting only when the maximal operator is bounded, since the Hajłasz gradient corresponds to $M(|\nabla f|)$.

The outline of the paper is as follows. We start by introducing our notation and basic definitions. Then we study Sobolev capacity and compare it with the Hausdorff measure. Next we derive existence of a quasicontinuous representative of generalized Orlicz function. Finally, we study relative capacity and compare it with the Sobolev capacity.

\section{Preliminaries}

We study spaces of functions defined in $\mathbb{R}^{n}$ or opens sets $\Omega \subset$ $\mathbb{R}^{n}$.

A real-valued function is $L$-almost increasing, $L \geqslant 1$, if $L f(s) \geqslant f(t)$ for $s>t$. So a 1 -almost increasing function is increasing. L-almost decreasing is defined analogously.

Definition 1. We say that $\varphi: \Omega \times[0, \infty) \longrightarrow[0, \infty]$ is a weak $\Phi$-function, and write $\varphi \in \Phi_{w}(\Omega)$, if the following conditions hold:

(i) For every $t \in[0, \infty)$ the function $x \longmapsto \varphi(x, t)$ is measurable and for every $x \in \Omega$ the function $t \longmapsto$ $\varphi(x, t)$ is non-decreasing and left-continuous.

(ii) $\varphi(x, 0)=\lim _{t \rightarrow 0^{+}} \varphi(x, t)=0$ and $\lim _{t \rightarrow \infty} \varphi(x, t)=$ $\infty$ for every $x \in \Omega$.

(iii) There exists $L \geqslant 1$ such that $t \longmapsto \varphi(x, t) / t$ is $L$-almost increasing in $(0, \infty)$, for every $x \in \Omega$.

If $\varphi \in \Phi_{w}(\Omega)$ is convex with respect to the second variable, then it is called a convex $\Phi$-function, and we write $\varphi \in \Phi_{c}(\Omega)$.

If there exists $\beta \in(0,1]$ such that $\varphi(x, \beta) \leqslant 1$ and $\varphi(x, 1 / \beta) \geqslant 1$ for all $x \in \Omega$, then we say that (A0) holds. Further, we say that $\varphi \in \Phi_{w}(\Omega)$ satisfies

(aInc) $p$ if there exists $L \geqslant 1$ such that $t \longmapsto \varphi(x, t) / t^{p}$ is $L$ almost increasing in $(0, \infty)$ for every $x \in \Omega$,

(aInc) if there exist $p>1$ such that $(\text { aInc })_{p}$ holds,

$(\mathrm{aDec})_{q}$ if there exists $L \geqslant 1$ such that $t \longmapsto \varphi(x, t) / t^{q}$ is $L$ almost decreasing in $(0, \infty)$ for every $x \in \Omega$,

$(\mathrm{aDec})$ if there exist $q>1$ such that $(\mathrm{aDec})_{q}$ holds.

The corresponding conditions with $L=1$ are denoted by (Inc) and (Dec). Note that the definition of weak $\Phi$-function includes assumption (aInc) $)_{1}$.

Definition 2. Let $\varphi \in \Phi_{w}(\Omega)$ and define the modular $\varrho_{\varphi(\cdot)}$ for $f \in L^{0}(\Omega)$ by

$$
\varrho_{\varphi(\cdot)}(f):=\int_{\Omega} \varphi(x,|f(x)|) d x .
$$

The generalized Orlicz space, also called Musielak-Orlicz space, is defined as the set

$$
L^{\varphi(\cdot)}(\Omega):=\left\{f \in L^{0}(\Omega): \lim _{\lambda \longrightarrow 0^{+}} \varrho_{\varphi(\cdot)}(\lambda f)=0\right\}
$$

equipped with the (Luxemburg) norm

$$
\|f\|_{\varphi(\cdot)}:=\inf \left\{\lambda>0: \varrho_{\varphi(\cdot)}\left(\frac{f}{\lambda}\right) \leqslant 1\right\} .
$$

For $\varphi \in \Phi_{c}(\Omega), L^{\varphi(\cdot)}(\Omega)$ is a Banach space [18, Theorem 2.3.13.].

Definition 3. A function $f \in L^{\varphi(\cdot)}(\Omega)$ belongs to Sobolev space $W^{1, \varphi(\cdot)}(\Omega)$, if its weak partial derivatives $\partial_{1} f, \ldots, \partial_{n} f$ exist and belong to $L^{\varphi(\cdot)}(\Omega)$, that is,

$$
W^{1, \varphi(\cdot)}(\Omega):=\left\{f \in W_{l o c}^{1,1}(\Omega): f,|\nabla f| \in L^{\varphi(\cdot)}(\Omega)\right\} .
$$

We define a semimodular on $W^{1, \varphi(\cdot)}(\Omega)$ by

$$
\varrho_{1, \varphi(\cdot)}(f):=\varrho_{\varphi(\cdot)}(f)+\varrho_{\varphi(\cdot)}(|\nabla f|) .
$$

which induces a quasinorm by $\|f\|_{1, \varphi(\cdot)}:=\inf \{\lambda>0$ : $\left.\varrho_{1, \varphi(\cdot)}(f / \lambda) \leqslant 1\right\}$.

Lemma 4. Let $\varphi \in \Phi_{c}(\Omega)$ satisfy (aInc) and (aDec), and let $f_{j}, g_{j} \in W^{1, \varphi(\cdot)}(\Omega)$ for $j=1,2, \ldots$ Assume further that the sequence $\left(\varrho_{1, \varphi(\cdot)}\left(g_{j}\right)\right)_{j=1}^{\infty}$ is bounded. If $\varrho_{1, \varphi(\cdot)}\left(f_{j}-g_{j}\right) \longrightarrow 0$ as $j \longrightarrow \infty$, then

$$
\left|\varrho_{1, \varphi(\cdot)}\left(f_{j}\right)-\varrho_{1, \varphi(\cdot)}\left(g_{j}\right)\right| \longrightarrow 0 \text { as } j \longrightarrow \infty .
$$

Proof. Since $\varphi$ is convex and satisfies (aDec) it satisfies (Dec) for some possible larger exponent $q$ by Lemma 2.6 of [13]. Let $\lambda \in(0,1)$. By convexity and (Dec) we obtain

$$
\begin{aligned}
\varphi\left(x,\left|f_{j}\right|\right)= & \varphi\left(x,\left|f_{j}-g_{j}+g_{j}\right|\right) \\
\leqslant & \varphi\left(x, \frac{\lambda}{\lambda}\left|f_{j}-g_{j}\right|+\frac{1-\lambda}{1-\lambda}\left|g_{j}\right|\right) \\
\leqslant & \lambda \varphi\left(x, \frac{1}{\lambda}\left|f_{j}-g_{j}\right|\right) \\
& +(1-\lambda) \varphi\left(x, \frac{1}{1-\lambda}\left|g_{j}\right|\right) \\
\leqslant & \lambda^{1-q} \varphi\left(x,\left|f_{j}-g_{j}\right|\right) \\
& +(1-\lambda)^{1-q} \varphi\left(x,\left|g_{j}\right|\right) .
\end{aligned}
$$

Next we subtract $\varphi\left(x, g_{j}\right)$ from both sides and integrate over $\Omega$. Hence

$$
\begin{aligned}
\varrho_{\varphi(\cdot)}\left(f_{j}\right)-\varrho_{\varphi(\cdot)}\left(g_{j}\right) \leqslant & \lambda^{1-q} \varrho_{\varphi(\cdot)}\left(f_{j}-g_{j}\right) \\
& +\left((1-\lambda)^{1-q}-1\right) \varrho_{\varphi(\cdot)}\left(g_{j}\right) .
\end{aligned}
$$

Note that the choice $\lambda=1 / 2$ implies that $\left(\varrho_{\varphi(\cdot)}\left(f_{j}\right)\right)$ is a bounded sequence. 
Swapping $f_{j}$ and $g_{j}$ gives a similar inequality, and, combining the inequalities, we find that

$$
\begin{aligned}
\mid \varrho_{\varphi(\cdot)} & \left(f_{j}\right)-\varrho_{\varphi(\cdot)}\left(g_{j}\right) \mid \\
\leqslant & \lambda^{1-q} \varrho_{\varphi(\cdot)}\left(f_{j}-g_{j}\right) d x \\
& \quad+\left((1-\lambda)^{1-q}-1\right)\left(\varrho_{\varphi(\cdot)}\left(f_{j}\right)+\varrho_{\varphi(\cdot)}\left(g_{j}\right)\right) .
\end{aligned}
$$

The same argument can be applied also to the weak gradient. Hence

$$
\begin{aligned}
&\left|\varrho_{1, \varphi(\cdot)}\left(f_{j}\right)-\varrho_{1, \varphi(\cdot)}\left(g_{j}\right)\right| \\
& \leqslant \lambda^{1-q} \varrho_{1, \varphi(\cdot)}\left(f_{j}-g_{j}\right) \\
& \quad+\left((1-\lambda)^{1-q}-1\right)\left(\varrho_{1, \varphi(\cdot)}\left(f_{j}\right)+\varrho_{1, \varphi(\cdot)}\left(g_{j}\right)\right) .
\end{aligned}
$$

Let $\varepsilon>0$ be given. Since $\varrho_{1, \varphi(\cdot)}\left(f_{j}\right)+\varrho_{1, \varphi(\cdot)}\left(g_{j}\right) \leqslant 2 c$, we can choose $\lambda$ so small that

$$
\left((1-\lambda)^{1-q}-1\right)\left(\varrho_{1, \varphi(\cdot)}\left(f_{j}\right)+\varrho_{1, \varphi(\cdot)}\left(g_{j}\right)\right) \leqslant \frac{\varepsilon}{2} .
$$

We can then choose $j_{0}$ so large that $\lambda^{1-q} \varrho_{1, \varphi(\cdot)}\left(f_{j}-g_{j}\right) \leqslant \varepsilon / 2$ when $j \geqslant j_{0}$ and it follows that $\left|\varrho_{1, \varphi(\cdot)}\left(f_{j}\right)-\varrho_{1, \varphi(\cdot)}\left(g_{j}\right)\right| \leqslant \varepsilon$.

The first claim of the following lemma has been proved in [16, Lemma 4.4]. The proof for the second claim is similar.

Lemma 5. Let $\varphi \in \Phi_{w}\left(\mathbb{R}^{n}\right)$ satisfy $(A 0)$ and let $E \subset \mathbb{R}^{n}$ be bounded.

(a) If $\varphi$ satisfies $(\text { aInc) })_{p}$, then $L^{\varphi(\cdot)}(E) \hookrightarrow L^{p}(E)$.

(b) If $\varphi$ satisfies $(a D e c)_{q}$, then $L^{q}(E) \hookrightarrow L^{\varphi(\cdot)}(E)$.

Lemma 6. Let $\Omega \subset \mathbb{R}^{n}$. Let $\varphi \in \Phi_{w}(\Omega)$ satisfy (aInc) $p$ and $(a D e c)_{q}$. Then

$$
\|f\|_{\varphi(\cdot)} \leqslant \max \left\{\left(L \varrho_{\varphi(\cdot)}(f)\right)^{1 / q},\left(L \varrho_{\varphi(\cdot)}(f)\right)^{1 / p}\right\}
$$

where $L$ is the maximum of the constants from (aInc) and (aDec).

Proof. If $\varrho_{\varphi(\cdot)}(f)=0$, then $\|f\|_{\varphi(\cdot)}=0$ and the claim holds.

Let $\varrho_{\varphi(\cdot)}(f)>0$ and assume first that $L \varrho_{\varphi(\cdot)}(f) \leqslant 1$.Then $(\mathrm{aDec})$ gives that

$$
\begin{aligned}
& \varphi\left(x, \frac{|f(x)|}{\left(L \varrho_{\varphi(\cdot)}(f)\right)^{1 / q}}\right) \\
& \leqslant L\left(L \varrho_{\varphi(\cdot)}(f)\right)^{-1} \varphi(x,|f(x)|) \\
& =\left(\varrho_{\varphi(\cdot)}(f)\right)^{-1} \varphi(x,|f(x)|) .
\end{aligned}
$$

Integrating over $\Omega$, we find that $\left.\varrho_{\varphi(\cdot)}\left(f /\left(L \varrho_{\varphi(\cdot)}(f)\right)^{1 / q}\right)\right) \leqslant 1$, which yields $\|f\|_{\varphi(\cdot)} \leqslant\left(L \varrho_{\varphi(\cdot)}(f)\right)^{1 / q}$. If $L \varrho_{\varphi(\cdot)}(f)>1$ we similarly use (aInc) to conclude that $\|f\|_{\varphi(\cdot)} \leqslant\left(L \varrho_{\varphi(\cdot)}(f)\right)^{1 / p}$. The claim follows from these two cases.

\section{Sobolev Capacity}

We define a set of test-functions for the capacity of the set $E$ by

$$
\begin{aligned}
& S_{1, \varphi(\cdot)}(E):=\left\{f \in W^{1, \varphi(\cdot)}\left(\mathbb{R}^{n}\right): f\right. \\
& \quad \geqslant 1 \text { in an open set containing } E \text { and } f \geqslant 0\} .
\end{aligned}
$$

The generalized Orlicz $\varphi(\cdot)$-capacity of $E$ is defined by

$$
C_{\varphi(\cdot)}(E):=\inf _{f \in S_{1, \varphi(\cdot)}(E)} \int_{\mathbb{R}^{n}} \varphi(x, f)+\varphi(x,|\nabla f|) d x .
$$

We now prove the following properties for the set function $E \longmapsto C_{\varphi(\cdot)}(E)$. We emphasize that these do not require the convexity of $\varphi$.

Proposition 7. Let $\varphi \in \Phi_{w}\left(\mathbb{R}^{n}\right)$.

(S1) $C_{\varphi(\cdot)}(\emptyset)=0$.

(S2) If $E_{1} \subset E_{2} \subset \mathbb{R}^{n}$, then $C_{\varphi(\cdot)}\left(E_{1}\right) \leqslant C_{\varphi(\cdot)}\left(E_{2}\right)$.

(S3) If $E \subset \mathbb{R}^{n}$, then $C_{\varphi(\cdot)}(E)=\inf _{U \supset E \text { open }} C_{\varphi(\cdot)}(U)$.

(S4) If $E_{1}, E_{2} \subset \mathbb{R}^{n}$, then $C_{\varphi(\cdot)}\left(E_{1} \cup E_{2}\right)+C_{\varphi(\cdot)}\left(E_{1} \cap E_{2}\right) \leqslant$ $C_{\varphi(\cdot)}\left(E_{1}\right)+C_{\varphi(\cdot)}\left(E_{2}\right)$.

(S5) If $K_{1} \supset K_{2} \supset \ldots$ are compact, then $\lim _{i \rightarrow \infty} C_{\varphi(\cdot)}\left(K_{i}\right)=$ $C_{\varphi(\cdot)}\left(\bigcap_{i=1}^{\infty} K_{i}\right)$.

Proof. (S1) follows from testing with $f=0$. Since a testfunction for $E_{2}$ is also a test-function for $E_{1}$, (S2) follows from the infimum in the definition of capacity.

If $U \supset E$, then $C_{\varphi(\cdot)}(E) \leqslant C_{\varphi(\cdot)}(U)$ by (S2). Thus,

$$
C_{\varphi(\cdot)}(E) \leqslant \inf _{U \supset E \text { open }} C_{\varphi(\cdot)}(U)
$$

For the opposite inequality, let $\varepsilon>0$ and choose $f \in S_{1, \varphi(\cdot)}(E)$ such that

$$
\varrho_{1, \varphi(\cdot)}(f) \leqslant C_{\varphi(\cdot)}(E)+\varepsilon
$$

Denote $V:=\operatorname{int}\{f \geqslant 1\}$. Then $f \in S_{1, \varphi(\cdot)}(V)$, and so

$$
\begin{aligned}
\inf _{U \supset \text { open }} C_{\varphi(\cdot)}(U) & \leqslant C_{\varphi(\cdot)}(V) \leqslant \varrho_{1, \varphi(\cdot)}(f) \\
& \leqslant C_{\varphi(\cdot)}(E)+\varepsilon .
\end{aligned}
$$

This implies $(\mathrm{S} 3)$ as $\varepsilon \longrightarrow 0^{+}$.

Let $\varepsilon>0$. Choose $f_{j} \in S_{1, \varphi(\cdot)}\left(E_{j}\right)$ such that

$$
\varrho_{1, \varphi(\cdot)}\left(f_{j}\right) \leqslant C_{\varphi(\cdot)}\left(E_{j}\right)+\varepsilon \quad \text { for } j \in\{1,2\} \text {. }
$$

Since $\max \left\{f_{1}, f_{2}\right\} \geqslant 1$ in an open set containing $E_{1} \cup E_{2}$, it follows that $\max \left\{f_{1}, f_{2}\right\} \in S_{1, \varphi(\cdot)}\left(E_{1} \cup E_{2}\right)$. Similarly, $\min \left\{f_{1}, f_{2}\right\} \in S_{1, \varphi(\cdot)}\left(E_{1} \cap E_{2}\right)$. 
The lattice property of Sobolev functions [18, Proposition 8.1.9] implies that $\nabla \max \left\{f_{1}, f_{2}\right\}=\nabla f_{1}$ and $\nabla \min \left\{f_{1}, f_{2}\right\}=$ $\nabla f_{2}$ almost everywhere in $A=\left\{f_{1} \geqslant f_{2}\right\}$. Therefore

$$
\begin{aligned}
\int_{A} \varphi & \left(x,\left|\nabla \max \left\{f_{1}, f_{2}\right\}\right|\right) d x \\
& +\int_{A} \varphi\left(x,\left|\nabla \min \left\{f_{1}, f_{2}\right\}\right|\right) d x \\
= & \int_{A} \varphi\left(x,\left|\nabla f_{1}\right|\right) d x+\int_{A} \varphi\left(x,\left|\nabla f_{2}\right|\right) d x .
\end{aligned}
$$

The same argument also gives the equality in the complement of $A$, i.e. $\left\{f_{1}<f_{2}\right\}$. Hence

$$
\begin{aligned}
\varrho_{\varphi(\cdot)} & \left(\nabla \max \left\{f_{1}, f_{2}\right\}\right)+\varrho_{\varphi(\cdot)}\left(\nabla \min \left\{f_{1}, f_{2}\right\}\right) \\
= & \varrho_{\varphi(\cdot)}\left(\nabla f_{1}\right)+\varrho_{\varphi(\cdot)}\left(\nabla f_{2}\right)
\end{aligned}
$$

almost everywhere. An analogous results holds without the derivative $\nabla$.

Since $\max \left\{f_{1}, f_{2}\right\} \in S_{1, \varphi(\cdot)}\left(E_{1} \cup E_{2}\right)$, it follows from the definition that $C_{\varphi(\cdot)}\left(E_{1} \cup E_{2}\right) \leqslant \varrho_{1, \varphi(\cdot)}\left(\max \left\{f_{1}, f_{2}\right\}\right)$, and similarly $C_{\varphi(\cdot)}\left(E_{1} \cap E_{2}\right) \leqslant \varrho_{1, \varphi(\cdot)}\left(\min \left\{f_{1}, f_{2}\right\}\right)$. Combining this with the conclusion of the previous paragraph, we find that

$$
\begin{aligned}
C_{\varphi(\cdot)} & \left(E_{1} \cup E_{2}\right)+C_{\varphi(\cdot)}\left(E_{1} \cap E_{2}\right) \\
& \leqslant \varrho_{1, \varphi(\cdot)}\left(\max \left\{f_{1}, f_{2}\right\}\right)+\varrho_{1, \varphi(\cdot)}\left(\min \left\{f_{1}, f_{2}\right\}\right) \\
& =\varrho_{1, \varphi(\cdot)}\left(f_{1}\right)+\varrho_{1, \varphi(\cdot)}\left(f_{2}\right) \\
& \leqslant C_{\varphi(\cdot)}\left(E_{1}\right)+C_{\varphi(\cdot)}\left(E_{2}\right)+2 \varepsilon .
\end{aligned}
$$

(S4) follows from this as $\varepsilon \longrightarrow 0^{+}$.

It remains to prove (S5). Since $\bigcap_{i=1}^{\infty} K_{i} \subset K_{j}$ for any $j$, the " $\geqslant$ "-inequality follows from (S2). To prove the opposite inequality, we choose an open $U \supset \bigcap_{i=1}^{\infty} K_{i}$. Since $\bigcap_{i=1}^{\infty} K_{i}$ is compact and $\left(K_{j}\right)$ is decreasing, there is a positive integer $k$ such that, $K_{i} \subset U$ for all $i \geqslant k$. Then, again by (S2), we have

$$
\lim _{i \rightarrow \infty} C_{\varphi(\cdot)}\left(K_{i}\right) \leqslant C_{\varphi(\cdot)}(U) .
$$

Taking the infimum of this inequality over such sets $U$, we obtain the claim by (S3).

Notice that we need convexity for the next property.

Theorem 8. Let $\varphi \in \Phi_{c}\left(\mathbb{R}^{n}\right)$ satisfy (aInc) and (aDec) and $E_{1} \subset E_{2} \subset \cdots \subset \mathbb{R}^{n}$. Then

$$
\text { (S6) } \lim _{i \rightarrow \infty} C_{\varphi(\cdot)}\left(E_{i}\right)=C_{\varphi(\cdot)}\left(\bigcup_{i=1}^{\infty} E_{i}\right) \text {. }
$$

Proof. Let us denote $E:=\bigcup_{i=1}^{\infty} E_{i}$. By (S2), $\lim _{i \rightarrow \infty} C_{\varphi(\cdot)}\left(E_{i}\right) \leqslant$ $C_{\varphi(\cdot)}(E)$.

Now to prove the opposite inequality, we may assume that $\lim _{i \rightarrow \infty} C_{\varphi(\cdot)}\left(E_{i}\right)<\infty$. Let $f_{i} \in S_{1, \varphi(\cdot)}\left(E_{i}\right)$ and $\varrho_{1, \varphi(\cdot)}\left(f_{i}\right) \leqslant$ $C_{\varphi(\cdot)}\left(E_{i}\right)+2^{-i}$ for every $i \in \mathbb{N}$.

The space $L^{\varphi(\cdot)}\left(\mathbb{R}^{n}\right)$ is uniformly convex and reflexive [20, Theorem 1.3]. The same holds for $W^{1, \varphi(\cdot)}$, which is a closed subspace of $\left(L^{\varphi(\cdot)}\right)^{n+1}$. By reflexivity, the bounded sequence $\left(f_{i}\right)$ has a subsequence which converges weakly to a function $f \in W^{1, \varphi(\cdot)}\left(\mathbb{R}^{n}\right)$. It follows from the Banach-Saks theorem that

$$
\frac{1}{m} \sum_{i=1}^{m} f_{i} \longrightarrow f \quad \text { in } W^{1, \varphi(\cdot)}\left(\mathbb{R}^{n}\right) \text { as } m \longrightarrow \infty .
$$

Let $g_{j}:=(1 / j(j-1)) \sum_{i=j+1}^{j^{2}} f_{i}$. Then

$$
\begin{aligned}
\frac{1}{j^{2}} \sum_{i=1}^{j^{2}} f_{i}-g_{j}= & \left(\frac{1}{j^{2}}-\frac{1}{j(j-1)}\right) \sum_{i=1}^{j^{2}} f_{i} \\
& +\frac{1}{j(j-1)} \sum_{i=1}^{j} f_{i}
\end{aligned}
$$

from which we get, by the triangle inequality, that

$$
\begin{aligned}
\left\|\frac{1}{j^{2}} \sum_{i=1}^{j^{2}} f_{i}-g_{j}\right\|_{1, \varphi(\cdot)} \leqslant & \frac{1}{j-1}\left\|\frac{1}{j^{2}} \sum_{i=1}^{j^{2}} f_{i}\right\|_{1, \varphi(\cdot)} \\
& +\frac{1}{j-1}\left\|\frac{1}{j} \sum_{i=1}^{j} f_{i}\right\|_{1, \varphi(\cdot)} \longrightarrow 0
\end{aligned}
$$

as $j \longrightarrow \infty$, so that $g_{j} \longrightarrow f$ in $W^{1, \varphi(\cdot)}\left(\mathbb{R}^{n}\right)$.

By the convexity of the modular and the choice of $g_{j}$, we obtain that

$$
\begin{aligned}
\varrho_{1, \varphi(\cdot)}\left(g_{j}\right) & \leqslant \frac{1}{j(j-1)} \sum_{i=j+1}^{j^{2}} \varrho_{1, \varphi(\cdot)}\left(f_{i}\right) \leqslant \sup _{i \geqslant j+1} \varrho_{1, \varphi(\cdot)}\left(f_{i}\right) \\
& \leqslant \sup _{i \geqslant j+1}\left(C_{\varphi(\cdot)}\left(E_{i}\right)+2^{-i}\right) .
\end{aligned}
$$

Now $C_{\varphi(\cdot)}\left(E_{i}\right)$ increases in $i$ whereas $2^{-i}$ decreases. Hence

$$
\varrho_{1, \varphi(\cdot)}\left(g_{j}\right) \leqslant \lim _{i \rightarrow \infty} C_{\varphi(\cdot)}\left(E_{i}\right)+2^{-j} .
$$

By considering a subsequence if necessary, we may assume that $\left\|g_{j+1}-g_{j}\right\|_{1, \varphi(\cdot)} \leqslant 2^{-j}$. Then $h_{j}:=g_{j}+\sum_{i=j}^{\infty} \mid g_{i+1}-$ $g_{i} \mid \in W^{1, \varphi(\cdot)}\left(\mathbb{R}^{n}\right)$. By definition of a test-function, there exists an open set $U_{i} \supset E_{i}$ such that $f_{i} \geqslant 1$ in $U_{i}$. As $E_{j}$ is an increasing sequence, it follows that $E_{j} \subset U_{i}$ for every $j \leqslant i$. In $\bigcap_{i=j+1}^{j^{2}} U_{j}, f_{i} \geqslant 1$ for all relevant $i$, so that $g_{j} \geqslant 1$ in the same (open) set. Since $h_{j} \geqslant \sup _{i \geqslant j} g_{j}$, we have

$$
E_{i} \subset \operatorname{int}\left\{g_{i} \geqslant 1\right\} \subset \operatorname{int}\left\{h_{j} \geqslant 1\right\} .
$$

Taking the union over $i$ of the previous inclusion, we find that $h_{j} \geqslant 1$ in the open set $\bigcup_{i=j}^{\infty} \operatorname{int}\left\{g_{j} \geqslant 1\right\} \supset E$, so that $h_{j} \in$ $S_{1, \varphi(\cdot)}(E)$. Hence

$$
C_{\varphi(\cdot)}(E) \leqslant \varrho_{1, \varphi(\cdot)}\left(h_{j}\right) \quad \text { for } j=1,2, \ldots
$$


Furthermore,

$$
\left\|h_{j}-g_{j}\right\|_{1, \varphi(\cdot)} \leqslant \sum_{i=j}^{\infty}\left\|g_{i+1}-g_{i}\right\|_{1, \varphi(\cdot)} \leqslant \sum_{i=j}^{\infty} 2^{-i}=2^{-j+1}
$$

and, hence, by [18, Corollary 2.1.15], $\varrho_{1, \varphi(\cdot)}\left(h_{j}-g_{j}\right) \leqslant \| h_{j}-$ $g_{j} \|_{1, \varphi(\cdot)} \longrightarrow 0$ as $j \longrightarrow \infty$. Then, using Lemma 4 , we also have $\left|\varrho_{1, \varphi(\cdot)}\left(h_{j}\right)-\varrho_{1, \varphi(\cdot)}\left(g_{j}\right)\right| \longrightarrow 0$ as $j \longrightarrow \infty$, which we apply in (31) to obtain

$$
C_{\varphi(\cdot)}(E) \leqslant \lim _{j \longrightarrow \infty} \varrho_{1, \varphi(\cdot)}\left(h_{j}\right)=\lim _{j \longrightarrow \infty} \varrho_{1, \varphi(\cdot)}\left(g_{j}\right)
$$

and further (29) implies

$$
\begin{aligned}
C_{\varphi(\cdot)}(E) & \leqslant \lim _{j \rightarrow \infty} \varrho_{1, \varphi(\cdot)}\left(g_{j}\right) \\
& \leqslant \lim _{j \longrightarrow \infty}\left(\lim _{i \rightarrow \infty} C_{\varphi(\cdot)}\left(E_{i}\right)+2^{-j}\right) \\
& =\lim _{i \longrightarrow \infty} C_{\varphi(\cdot)}\left(E_{i}\right) .
\end{aligned}
$$

In the next result we use a trick to get back to weak $\Phi$ functions despite an application of (S6).

Theorem 9. Let $\varphi \in \Phi_{w}\left(\mathbb{R}^{n}\right)$ satisfy (aInc) and (aDec) and $E_{1}, E_{2}, \ldots \subset \mathbb{R}^{n}$. Then

$$
\text { (S7) } C_{\varphi(\cdot)}\left(\bigcup_{i=1}^{\infty} E_{i}\right) \leqslant \sum_{i=1}^{\infty} C_{\varphi(\cdot)}\left(E_{i}\right) \text {. }
$$

Proof. Let $\psi \in \Phi_{c}\left(\mathbb{R}^{n}\right)$ with $\varphi \simeq \psi$ [21, Lemma 3.1]. Since $\varphi$ satisfies $(\mathrm{aDec}) \varphi \simeq \psi$ yields $\varphi \approx \psi$. Denote $F_{k}^{m}:=\cup_{i=m}^{k} E_{i}$ for $k=m, m+1, \ldots$ By induction on (S4), we obtain that

$$
\begin{aligned}
C_{\varphi(\cdot)}\left(F_{k}^{m}\right) & =C_{\varphi(\cdot)}\left(\bigcup_{i=m}^{k} E_{i}\right) \leqslant \sum_{i=m}^{k} C_{\varphi(\cdot)}\left(E_{i}\right) \\
& \leqslant \sum_{i=m}^{\infty} C_{\varphi(\cdot)}\left(E_{i}\right) .
\end{aligned}
$$

The same inequality holds also for $\psi$. Now, using (S6) for $\left(F_{k}\right)$, we have

$$
\begin{aligned}
C_{\psi(\cdot)}\left(\bigcup_{k=m}^{\infty} E_{k}\right) & =C_{\psi(\cdot)}\left(\bigcup_{k=m}^{\infty} F_{k}^{m}\right)=\lim _{k \rightarrow \infty} C_{\psi(\cdot)}\left(F_{k}^{m}\right) \\
& \leqslant \sum_{i=m}^{\infty} C_{\psi(\cdot)}\left(E_{i}\right)
\end{aligned}
$$

Furthermore, for $k=1,2, \ldots$, by (S4) and $\varphi \approx \psi$,

$$
\begin{aligned}
C_{\varphi(\cdot)}\left(\bigcup_{j=1}^{\infty} E_{j}\right) & \leqslant C_{\varphi(\cdot)}\left(F_{k}^{1}\right)+C_{\varphi(\cdot)}\left(\bigcup_{j=k+1}^{\infty} E_{j}\right) \\
& \leqslant C_{\varphi(\cdot)}\left(F_{k}^{1}\right)+c C_{\psi(\cdot)}\left(\bigcup_{j=k+1}^{\infty} E_{j}\right) .
\end{aligned}
$$

By the two estimates in the previous paragraph, we obtain that

$$
C_{\varphi(\cdot)}\left(\bigcup_{j=1}^{\infty} E_{j}\right) \leqslant \sum_{i=1}^{\infty} C_{\varphi(\cdot)}\left(E_{i}\right)+c \sum_{i=k}^{\infty} C_{\psi(\cdot)}\left(E_{i}\right) .
$$

Since the sum is finite (otherwise, there is nothing to prove), the second term on the right hand side tends to zero as $k \longrightarrow$ $\infty$. This gives the claim.

Remark 10. A set function satisfying the properties (S1), (S2), and (S7) is called an outer measure. This holds if $\varphi \in \Phi_{w}(\Omega)$ is satisfies (aInc) and (aDec). If $\varphi$ is convex and satisfies (aInc) and $(\mathrm{aDec})$, then it is a Choquet capacity, [22], i.e., it satisfies (S1), (S2), (S5), and (S6). Then, for every Borel set $E \subset \Omega$,

$$
\begin{aligned}
& C_{\varphi(\cdot)}(E) \\
& \quad=\sup \left\{C_{\varphi(\cdot)}(K): K \text { is compact and } K \subset E\right\} .
\end{aligned}
$$

\section{Sobolev Capacity and Hausdorff Measure}

In this section, we discuss simple relations between the generalized Orlicz capacity and the Lebesgue and Hausdorff measures.

Lemma 11. Let $\varphi \in \Phi_{w}\left(\mathbb{R}^{n}\right)$ satisfy (aDec) and (A0). Then every measurable set $E \subset \mathbb{R}^{n}$ satisfies $|E| \leqslant c C_{\varphi(\cdot)}(E)$, where $c>1$ depends on $(\mathrm{aDec})$ and $(\mathrm{AO})$.

Proof. Let $f \in S_{1, \varphi(\cdot)}(E)$. By (aDec), with exponent $q$ and constant $L$, we conclude that $\varphi(x, f(x) / \beta) \leqslant L \beta^{-q} \varphi(x, f(x))$. We have $\varphi(x, f(x) / \beta) \geqslant 1$ for $f(x) \geqslant 1$ by (A0). Therefore

$$
\begin{aligned}
|E| & \leqslant \int_{\mathbb{R}^{n}} \varphi\left(x, \frac{f}{\beta}\right) d x \leqslant L \beta^{-q} \int_{\mathbb{R}^{n}} \varphi(x, f) d x \\
& \leqslant L \beta^{-q} \varrho_{1, \varphi(\cdot)}(f) .
\end{aligned}
$$

Taking infimum over $f \in S_{1, \varphi(\cdot)}(E)$, we obtain the claim.

Proposition 12. Let $\varphi \in \Phi_{w}\left(\mathbb{R}^{n}\right)$ satisfy $(A 0),(\text { IInc })_{p}, p>1$, and $(a D e c)$. If $C_{\varphi(\cdot)}(E)=0$, then $C_{p}(E)=0$.

Proof. Let $B=B(0, R+1)$ and $\eta \in C_{0}^{\infty}(B)$ be a cut-off function with $\eta=1$ in $B(0, R), 0 \leqslant \eta \leqslant 1$ and $|\nabla \eta| \leqslant 2$. Let $f \in$ $L^{\varphi(\cdot)}\left(\mathbb{R}^{n}\right)$. By Lemma 5 , we obtain

$$
\|f \eta\|_{L^{p}\left(\mathbb{R}^{n}\right)}=\|f \eta\|_{L^{p}(B)} \leqslant c\|\eta f\|_{L^{\varphi(\cdot)}(B)} \leqslant c\|f\|_{L^{\varphi(\cdot)}\left(\mathbb{R}^{n}\right)}
$$

where $\mathrm{c}$ depends on (aInc) and $|B|$. The same inequality holds for $|\nabla f|$. Now $\nabla(f \eta)=\eta \nabla f+f \nabla \eta$, and, thus $|\nabla(f \eta)| \leqslant|\nabla f|+$ $2|f|$ by the assumptions on $\eta$. Since $\|f\|_{W^{1, \varphi(\cdot)}\left(\mathbb{R}^{n}\right)} \approx\|f\|_{L^{p}\left(\mathbb{R}^{n}\right)}+$ $\|\nabla f\|_{L^{p}\left(\mathbb{R}^{n}\right)}$ we obtain that

$$
\begin{aligned}
\|f \eta\|_{W^{1, p}\left(\mathbb{R}^{n}\right)} & \leq\|f \eta\|_{L^{p}\left(\mathbb{R}^{n}\right)}+\|\nabla(f \eta)\|_{L^{p}\left(\mathbb{R}^{n}\right)} \\
& \leq\|f\|_{W^{1, \varphi(\cdot)}\left(\mathbb{R}^{n}\right)} .
\end{aligned}
$$

Since $C_{\varphi(\cdot)}(E)=0$, we can choose $f_{i} \in S_{1, \varphi(\cdot)}(E \cap$ $B(0, R))$ with $\varrho_{1, \varphi(\cdot)}\left(f_{i}\right) \longrightarrow 0$. Since $\varphi$ satisfies (aDec), 
$\left\|f_{i}\right\|_{W^{1, \varphi(\cdot)}\left(\mathbb{R}^{n}\right)} \longrightarrow 0[18$, Lemma 2.1.11]. Thus the above inequality implies that $\left\|f_{i} \eta\right\|_{W^{1, p}\left(\mathbb{R}^{n}\right)} \longrightarrow 0$. Since $f_{i} \eta$ is a testfunction for the $p$-capacity of $E \cap B(0, R)$, we get $C_{p}(E \cap$ $B(0, R))=0$, for every $R>0$. Since $E=\bigcup_{i=1}^{\infty}(E \cap B(0, i))$, the claim follows by the subadditivity of the $p$-capacity.

In the previous result the assumption (aInc) is natural, since $p$ gives the capacity to compare with. However, the assumption $(\mathrm{aDec})$ is surprising. The next example shows that it is nevertheless needed.

Example 13. Let $\varphi(t)=0$ when $t \in[0,11 / 10]$ and $\varphi(t)=$ $t-11 / 10$ when $t>11 / 10$. Then $\varphi$ satisfies (aInc) with $p=1$ and (A0) with $\beta=10 / 21$. Let $B$ be an open ball with a radius one. Let $f$ be a Lipschitz-continuous function that is one in $B$, zero in $\mathbb{R}^{n} \backslash 2 B$ and linear in $2 B \backslash B$. Then $|\nabla f|=1$ in $2 B \backslash B$ and zero elsewhere. We obtain $\varrho_{1, \varphi}(f)=0$ and thus $C_{\varphi(\cdot)}(B)=0$. On the other hand $C_{p}(B)>0$.

The s-dimensional Hausdorff measure of a set $E \subset \mathbb{R}^{n}$ is denoted by $\mathscr{H}^{s}(E)$.

Corollary 14. Let $\varphi \in \Phi_{w}\left(\mathbb{R}^{n}\right)$ satisfy $(A 0),(\text { aInc })_{p}$ with $p>1$ and $(a D e c)$.

(1) If $p \leqslant n$ and $E \subset \mathbb{R}^{n}$ with $C_{\varphi(\cdot)}(E)=0$, then $\mathscr{H}^{s}(E)=0$ for all $s>n-p$.

(2) If $p>n$, then $C_{\varphi(\cdot)}(E)=0$ if and only if $E=\emptyset$, where $E \subset \mathbb{R}^{n}$.

Proof. If $C_{\varphi(\cdot)}(E)=0$, we then obtain $C_{p}(E)=0$ by Proposition 12. This leads to the first claim by [23, Theorem 4, p. 156].

If $p>n$, then we may choose $s=0$ in (1) and so $\mathscr{H}^{0}(E)=$ 0 . Since $\mathscr{H}^{0}$ is a counting measure, $E$ must be an empty set. On the other hand, $C_{\varphi(\cdot)}(\emptyset)=0$, by $(\mathrm{S} 1)$.

Corollary 15. Let $\psi \in \Phi_{w}\left(\mathbb{R}^{n}\right)$ satisfy (A0), (aInc), and $(a D e c)_{q}$. Let $E \subset \mathbb{R}^{n}$ be bounded. If $C_{q}(E)=0$ or $\mathscr{H}^{n-q}(E)<$ $\infty$, then $C_{\psi(\cdot)}(E)=0$.

Proof. Let $C_{q}(E)=0$. By Lemma $5, L^{q}(E) \hookrightarrow L^{\psi(\cdot)}(E)$. The remaining proof follows the same procedure as in the proof of Proposition 12. If $\mathscr{H}^{n-q}(E)<\infty$, it follows from [23, Theorem 3, p. 154] that $C_{q}(E)=0$ and thus the claim follows from the first part.

\section{Quasicontinuity}

In this section, we prove the existence of $\varphi(\cdot)$-quasicontinuous representatives of generalized Orlicz functions. A function $f: \mathbb{R}^{n} \longrightarrow[-\infty, \infty]$ is $\varphi(\cdot)$-quasicontinuous if for every $\varepsilon>0$ there exists an open set $U$ with $C_{\varphi(\cdot)}(U)<\varepsilon$ such that $f$ restricted to $\mathbb{R}^{n} \backslash U$ is continuous. We say that a claim holds $\varphi(\cdot)$-quasieverywhere if it holds except in a set of Sobolev $\varphi(\cdot)$-capacity zero.

In this section we assume the density of continuous functions. A sufficient condition for this can be found in Theorem 6.5 of [16].
Lemma 16. Let $\varphi \in \Phi_{w}\left(\mathbb{R}^{n}\right)$ satisfy (aInc) and (aDec). Then, for each Cauchy sequence $C\left(\mathbb{R}^{n}\right) \cap W^{1, \varphi(\cdot)}\left(\mathbb{R}^{n}\right)$, there is a subsequence which converges pointwise $\varphi(\cdot)$-quasieverywhere in $\mathbb{R}^{n}$. Moreover, the convergence is uniform outside a set of arbitrarily small Sobolev $\varphi(\cdot)$-capacity.

Proof. Let $\left(f_{i}\right)$ be a Cauchy sequence in $C\left(\mathbb{R}^{n}\right) \cap W^{1, \varphi(\cdot)}\left(\mathbb{R}^{n}\right)$. We assume without loss of generality, by considering a subsequence if necessary, that $\left\|f_{i}-f_{i+1}\right\|_{1, \varphi(\cdot)} \leqslant 4^{-i}$ for every $i=1,2, \ldots$ For $i \in \mathbb{N}$ we denote $g_{i}:=2^{i}\left|f_{i}-f_{i+1}\right| \in$ $W^{1, \varphi(\cdot)}\left(\mathbb{R}^{n}\right), E_{i}:=\left\{g_{i}>1\right\}$ and $F_{j}:=\bigcup_{i=j}^{\infty} E_{i}$. Note that $\left\|g_{i}\right\|_{1, \varphi(\cdot)} \leqslant 2^{-i}$. We obtain by Corollary 2.1.15(a) of [18] that

$$
C_{\varphi(\cdot)}\left(E_{i}\right) \leqslant \varrho_{1, \varphi(\cdot)}\left(g_{i}\right) \leqslant\left\|g_{i}\right\|_{1, \varphi(\cdot)} \leqslant 2^{-i} .
$$

The subadditivity (S7) further implies

$$
C_{\varphi(\cdot)}\left(F_{j}\right) \leqslant \sum_{i=j}^{\infty} C_{\varphi(\cdot)}\left(E_{i}\right) \leqslant \sum_{i=j}^{\infty} 2^{-i}=2^{1-j} .
$$

Since $\left(F_{j}\right)_{j=1}^{\infty}$ is decreasing and $C_{\varphi(\cdot)}\left(F_{j}\right) \geqslant 0$, the limit $\lim _{j \rightarrow \infty} C_{\varphi(\cdot)}\left(F_{j}\right)$ exists. Since $\bigcap_{j=1}^{\infty} F_{j} \subset F_{k}$ for all $k=1,2, \ldots$, we obtain by (S2) that

$$
C_{\varphi(\cdot)}\left(\bigcap_{j=1}^{\infty} F_{j}\right) \leqslant \lim _{j \rightarrow \infty} C_{\varphi(\cdot)}\left(F_{j}\right) \leqslant \lim _{j \longrightarrow \infty} 2^{1-j}=0 .
$$

Now $\left(f_{j}\right)$ converges pointwise in $\mathbb{R}^{n} \backslash \bigcap_{j=1}^{\infty} F_{j}$ and $C_{\varphi(\cdot)}\left(\bigcap_{j=1}^{\infty} F_{j}\right)=0$, so the first claim of the lemma is proved. Moreover, for $x \in \mathbb{R}^{n} \backslash F_{j}$ and $k>l>j$,

$$
\begin{aligned}
\left|f_{l}(x)-f_{k}(x)\right| & \leqslant \sum_{i=l}^{k-1}\left|f_{i}(x)-f_{i+1}(x)\right| \leqslant \sum_{i=l}^{k-1} 2^{-i} \\
& <2^{1-l} .
\end{aligned}
$$

Therefore, the convergence is uniform in $\mathbb{R}^{n} \backslash F_{j}$, and the second claim follows.

The existence of the so-called $\varphi(\cdot)$-quasicontinuous representative follows from Lemma 16 by standard arguments (e.g., [18, Theorem 11.1.3]):

Theorem 17. Let $\varphi \in \Phi_{w}\left(\mathbb{R}^{n}\right)$ satisfy (aInc) and (aDec). Assume that $C\left(\mathbb{R}^{n}\right) \cap W^{1, \varphi(\cdot)}\left(\mathbb{R}^{n}\right)$ is dense in $W^{1, \varphi(\cdot)}\left(\mathbb{R}^{n}\right)$. Then for each $f \in W^{1, \varphi(\cdot)}\left(\mathbb{R}^{n}\right)$, there exists a $\varphi(\cdot)$-quasicontinuous function $g \in W^{1, \varphi(\cdot)}\left(\mathbb{R}^{n}\right)$ such that $f=g$ almost everywhere in $\mathbb{R}^{n}$.

Lemma 18. Let $\varphi \in \Phi_{w}\left(\mathbb{R}^{n}\right)$ satisfy (aDec). Suppose that $f \in$ $W^{1, \varphi(\cdot)}\left(\mathbb{R}^{n}\right)$ is a nonnegative $\varphi(\cdot)$-quasicontinuous function with $f \geqslant 1$ in $E \subset \mathbb{R}^{n}$. Then for every $\varepsilon>0$ there exists $g \in S_{1, \varphi(\cdot)}(E)$ such that $\varrho_{1, \varphi(\cdot)}(f-g)<\varepsilon$.

Proof. Let $\varepsilon \in(0,1)$ and $\delta:=\varepsilon / 2 L 2^{q}\left(1+L \varrho_{1, \varphi(\cdot)}(f)\right)$, and let $U \subset \mathbb{R}^{n}$ be an open set such that $f$ restricted to $\mathbb{R}^{n} \backslash U$ 
is continuous and $C_{\varphi(\cdot)}(U)<\delta$. Moreover, let us take $h \in$ $S_{1, \varphi(\cdot)}(U)$ such that $\varrho_{1, \varphi(\cdot)}(h)<\delta$, and denote $g:=(1+\delta) f+h$. Then $g \in W^{1, \varphi(\cdot)}\left(\mathbb{R}^{n}\right)$. The set $V:=\left\{x \in \mathbb{R}^{n} \backslash U:(1+\delta) f(x)>\right.$ $1\} \cup U$ is open and contains $E$. Since $g \geqslant 1$ in $V, g \in S_{1, \varphi(\cdot)}(E)$. It remains to estimate $\varrho_{1, \varphi(\cdot)}(f-g)$.

Now, $\varphi(x, h+\delta f) \leqslant \varphi(x, 2 h)+\varphi(x, 2 \delta f)$. By $(\mathrm{aDec})_{p}$ and (aInc) ${ }_{1}$ we have $\varphi(x, 2 \delta f) \leqslant L 2^{q} \varphi(x, \delta f) \leqslant L^{2} 2^{q} \delta \varphi(x, f)$, and so $\varphi(x, h+\delta f) \leqslant L 2^{q}(\varphi(x, h)+L \delta \varphi(x, f))$. Therefore

$$
\begin{aligned}
\varrho_{\varphi(\cdot)}(f-g) & =\varrho_{\varphi(\cdot)}(h+\delta f) \\
& \leqslant L 2^{q}\left(\varrho_{\varphi(\cdot)}(h)+L \delta \varrho_{\varphi(\cdot)}(f)\right) \\
& <L 2^{q}\left(1+L \varrho_{\varphi(\cdot)}(f)\right) \delta=\frac{1}{2} \varepsilon
\end{aligned}
$$

by the assumption on $\delta$. An analogous inequality holds for the gradient, and so the claim follows.

\section{Relative Capacity}

In this section, we introduce relative $\varphi(\cdot)$-capacity, analogous to the relative $p(\cdot)$-capacity of variable exponent spaces in [18], taken with respect to an open set, $\Omega$, in $\mathbb{R}^{n}$.

Definition 19. Let $\Omega \subset \mathbb{R}^{n}, K \subset \Omega$ be compact, and $\varphi \in$ $\Phi_{w}(\Omega)$. Denote

$$
\begin{aligned}
& R_{\varphi(\cdot)}(K, \Omega):=\left\{f \in W^{1, \varphi(\cdot)}(\Omega) \cap C_{0}(\Omega): f\right. \\
& >1 \text { in } K \text { and } f \geqslant 0\} .
\end{aligned}
$$

We define $\operatorname{cap}_{\varphi(\cdot)}^{*}(K, \Omega):=\inf _{f \in R_{\varphi(\cdot)}(K, \Omega)} \varrho_{\varphi(\cdot)}(|\nabla f|)$. Further, for $U \subset \Omega$ open, we set

$$
\operatorname{cap}_{\varphi(\cdot)}(U, \Omega):=\sup _{K \subset U \text { compact }} \operatorname{cap}_{\varphi(\cdot)}^{*}(K, \Omega),
$$

and, for an arbitrary set $E \subset \Omega$, we define

$$
\operatorname{cap}_{\varphi(\cdot)}(E, \Omega):=\inf _{U \supset E \text { open }} \operatorname{cap}_{\varphi(\cdot)}(U, \Omega) .
$$

The number $\operatorname{cap}_{\varphi(\cdot)}(E, \Omega)$ is called the relative $\varphi(\cdot)$-capacity of $E$ with respect to $\Omega$.

In the next result we offer two alternate set of assumptions. It seems that $(\mathrm{aDec})$ alone is not sufficient, although we have not been able to prove this.

Proposition 20. If $\varphi \in \Phi_{w}(\Omega)$ satisfies either (Dec) or (A0), then

$$
\operatorname{cap}_{\varphi(\cdot)}^{*}(K, \Omega)=\inf _{f \in \tilde{R}_{\varphi(\cdot)}(K, \Omega)} \int_{\Omega} \varphi(x,|\nabla f|) d x,
$$

where $\widetilde{R}_{\varphi(\cdot)}(K, \Omega):=\left\{f \in W^{1, \varphi(\cdot)}(\Omega) \cap C_{0}(\Omega): f \geqslant 1\right.$ in $K$ and $f \geqslant 0\}$.

Proof. Since $R_{\varphi(\cdot)}(K, \Omega) \subset \widetilde{R}_{\varphi(\cdot)}(K, \Omega)$, the inequality " $\geqslant$ " is clear. Now, to prove the opposite inequality, we fix $\varepsilon>0$ and let $f \in \widetilde{R}_{\varphi(\cdot)}(K, \Omega)$, be such that

$$
\varrho_{\varphi(\cdot)}(|\nabla f|) \leqslant \inf _{g \in \widetilde{R}_{\varphi(\cdot)}(K, \Omega)} \varrho_{\varphi(\cdot)}(|\nabla g|)+\varepsilon .
$$

If (Dec) holds, we set $g:=(1+\varepsilon) f$. Then $g>1$ in $K$, so that

$$
\begin{aligned}
\operatorname{cap}_{\varphi(\cdot)}^{*}(K, \Omega) & \leqslant \varrho_{\varphi(\cdot)}(\nabla g)=\int_{\Omega} \varphi(x,(1+\varepsilon) \nabla f) d x \\
& \leqslant(1+\varepsilon)^{q} \int_{\Omega} \varphi(x,|\nabla f|) d x
\end{aligned}
$$

where we used (Dec) for the last inequality. The claim follows as $\varepsilon \longrightarrow 0^{+}$.

If, on the other hand, (A0) holds, then we fix $\eta \in C_{0}^{\infty}(\Omega)$ with $\eta=1$ in $\operatorname{supp} f$. Let $\lambda=\beta\|\nabla \eta\|_{\infty}^{-1}$. We set $g:=f+\varepsilon^{\prime} \lambda \eta \epsilon$ $R_{\varphi(\cdot)}(K, \Omega)$. Since the supports of $\nabla f$ and $\nabla \eta$ are disjoint, we observe that

$$
\begin{aligned}
\varrho_{\varphi(\cdot)}(\nabla g) & =\varrho_{\varphi(\cdot)}(\nabla f)+\varrho_{\varphi(\cdot)}\left(\varepsilon^{\prime} \lambda \nabla \eta\right) \\
& \leqslant \varrho_{\varphi(\cdot)}(\nabla f)+L \varepsilon^{\prime} \varrho_{\varphi(\cdot)}(\lambda \nabla \eta),
\end{aligned}
$$

where $(\mathrm{aInc})_{1}$ has been used in the last inequality. The claim follows as $\varepsilon, \varepsilon^{\prime} \longrightarrow 0^{+}$.

Next, we show that $\operatorname{cap}_{\varphi(\cdot)}^{*}(K, \Omega)$ and $\operatorname{cap}_{\varphi(\cdot)}(K, \Omega)$ are the same; that is, the relative capacity is well defined on compact sets.

Proposition 21. Let $\varphi \in \Phi_{w}(\Omega)$. Then $\operatorname{cap}_{\varphi(\cdot)}^{*}(K, \Omega)=$ $\operatorname{cap}_{\varphi(\cdot)}(K, \Omega)$ for every compact $K \subset \Omega$.

Proof. The inequality cap $\cos _{(\cdot)}^{*}(K, \Omega) \leqslant \operatorname{cap}_{\varphi(\cdot)}(K, \Omega)$ follows directly from the definition.

Now, to prove the opposite inequality, fix $\varepsilon>0$ and let $f \in R_{\varphi(\cdot)}(K, \Omega)$ be such that $\varrho_{\varphi(\cdot)}(\nabla f) \leqslant \operatorname{cap}_{\varphi(\cdot)}^{*}(K, \Omega)+\varepsilon$. Then, $f$ is greater than one in $U:=f^{-1}(1, \infty)$, which is open since $f \in C_{0}(\Omega)$, and contains $K$. Thus, $f$ is also a valid testfunction for every compact set $K^{\prime} \subset U$, so that

$$
\begin{aligned}
\operatorname{cap}_{\varphi(\cdot)}(U, \Omega) & =\sup _{K^{\prime} \subset U} \operatorname{cap}_{\varphi(\cdot)}^{*}\left(K^{\prime}, \Omega\right) \leqslant \varrho_{\varphi(\cdot)}(\nabla f) \\
& \leqslant \operatorname{cap}_{\varphi(\cdot)}^{*}(K, \Omega)+\varepsilon .
\end{aligned}
$$

The result follows from this as $\varepsilon \longrightarrow 0^{+}$.

Next, we show that the relative capacity has the same basic properties as the Sobolev capacity.

Proposition 22. Let $\Omega \subset \mathbb{R}^{n}$ be open and $\varphi \in \Phi_{w}\left(\mathbb{R}^{n}\right)$.

(R1) $\operatorname{cap}_{\varphi(\cdot)}(\emptyset, \Omega)=0$.

(R2) If $E \subset E^{\prime} \subset \Omega^{\prime} \subset \Omega$, then $\operatorname{cap}_{\varphi(\cdot)}(E, \Omega) \leqslant$ $\operatorname{cap}_{\varphi(\cdot)}\left(E^{\prime}, \Omega^{\prime}\right)$.

(R3) If $E \subset \Omega$, then $\operatorname{cap}_{\varphi(\cdot)}(E, \Omega)=\inf _{U \supset E \text { open }} \operatorname{cap}_{\varphi(\cdot)}(U$, $\Omega)$.

(R4) If $E, F \subset \Omega$, then

$$
\begin{gathered}
\operatorname{cap}_{\varphi(\cdot)}(E \cup F, \Omega)+\operatorname{cap}_{\varphi(\cdot)}(E \cap F, \Omega) \\
\quad \leqslant \operatorname{cap}_{\varphi(\cdot)}(E, \Omega)+\operatorname{cap}_{\varphi(\cdot)}(F, \Omega) .
\end{gathered}
$$


(R5) If $K_{1} \supset K_{2} \supset \cdots$ are compact, then $\lim _{i \rightarrow \infty} \operatorname{cap}_{\varphi(\cdot)}\left(K_{i}\right.$, $\Omega)=\operatorname{cap}_{\varphi(\cdot)}\left(\bigcap_{i=1}^{\infty} K_{i}, \Omega\right)$.

Proof. Properties (R1) and (R3) follow immediately from the definition. For proving property (R2), we observe that if $E^{\prime} \subset$ $U \subset \Omega^{\prime}$ then also $E \subset U \subset \Omega$. Thus

$$
\begin{aligned}
\operatorname{cap}_{\varphi(\cdot)}\left(E^{\prime}, \Omega^{\prime}\right) & =\inf _{E^{\prime} \subset U \subset \Omega^{\prime}} \operatorname{cap}_{\varphi(\cdot)}\left(U, \Omega^{\prime}\right) \\
& \geqslant \operatorname{cap}_{\varphi(\cdot)}(E, \Omega) .
\end{aligned}
$$

Properties (R4) and (R5) are proved exactly like (S4) and (S5).

The proof of the following results follows from (R4) by standard arguments, see, e.g., [18, Lemma 10.2.5].

Lemma 23. Suppose that $\varphi \in \Phi_{w}(\Omega)$ and $E_{1}, E_{2}, \ldots, E_{k} \subset \Omega$. Then,

$$
\begin{gathered}
\operatorname{cap}_{\varphi(\cdot)}\left(\bigcup_{i=1}^{k} E_{i}, \Omega\right)-\operatorname{cap}_{\varphi(\cdot)}\left(\bigcup_{i=1}^{k} A_{i}, \Omega\right) \\
\leqslant \sum_{i=1}^{k}\left(\operatorname{cap}_{\varphi(\cdot)}\left(E_{i}, \Omega\right)-\operatorname{cap}_{\varphi(\cdot)}\left(A_{i}, \Omega\right)\right),
\end{gathered}
$$

whenever $A_{i} \subset E_{i}, i=1,2, \ldots, k$ and $\operatorname{cap}_{\varphi(\cdot)}\left(\bigcup_{i=1}^{k} E_{i}, \Omega\right)<\infty$.

Furthermore, the previous lemma implies directly properties (R6) and (R7), see, e.g., [18, Theorem 10.2.6]. Note that these properties hold for the relative capacity without any extra assumptions on $\varphi$.

Theorem 24. Let $\varphi \in \Phi_{w}(\Omega)$.

(R6) If $E_{1} \subset E_{2} \subset \cdots \subset \Omega$, then $\lim _{i \rightarrow \infty} \operatorname{cap}_{\varphi(\cdot)}\left(E_{i}, \Omega\right)=$ $\operatorname{cap}_{\varphi(\cdot)}\left(\bigcup_{i=1}^{\infty} E_{i}, \Omega\right)$.

(R7) If $E_{i} \subset \Omega, i=1,2, \ldots$, then $\operatorname{cap}_{\varphi(\cdot)}\left(\bigcup_{i=1}^{\infty} E_{i}, \Omega\right) \leqslant$ $\sum_{i=1}^{\infty} \operatorname{cap}_{\varphi(\cdot)}\left(E_{i}, \Omega\right)$.

Remark 25. A set function satisfying the properties (R1), (R2), (R5), and (R6) is called a Choquet capacity [22]. They imply for every Borel set $E \subset \Omega$ that

$$
\begin{aligned}
& \operatorname{cap}_{\varphi(\cdot)}(E, \Omega) \\
& \quad=\sup \left\{\operatorname{cap}_{\varphi(\cdot)}(K, \Omega): K \text { is compact and } K \subset E\right\} .
\end{aligned}
$$

\section{Relationship between Capacities}

Lemma 26. Assume that $\varphi \in \Phi_{w}\left(\mathbb{R}^{n}\right)$ satisfies (A0) and $(a D e c)_{q}$. If $\Omega$ is bounded and $K \subset \Omega$ is compact, then,

$$
C_{\varphi(\cdot)}(K) \leqslant C \max \left\{\operatorname{cap}_{\varphi(\cdot)}(K, \Omega)^{1 / q}, \operatorname{cap}_{\varphi(\cdot)}(K, \Omega)\right\}
$$

where the constant $C$ depends on the dimension, $|\Omega|$ and the constants in $(\mathrm{AO})$ and $(\mathrm{aDec})$.
Proof. We may assume that $\operatorname{cap}_{\varphi(\cdot)}(K, \Omega)<\infty$. Let $f \in$ $R_{\varphi(\cdot)}(K, \Omega)$ with $\varrho_{\varphi(\cdot)}(|\nabla f|)<2 \operatorname{cap}_{\varphi(\cdot)}(K, \Omega)$. Extend $f$ by zero outside of $\Omega$ and set $g:=\min \{1, f\}$. Since $f \in C_{0}(\Omega)$ and $f>1$ in the compact set $K, U=\{f>1\} \supset K$ is open. Hence $g \in S_{1, \varphi(\cdot)}(K)$ and so

$$
\begin{aligned}
C_{\varphi(\cdot)}(K) & \leqslant \int_{\mathbb{R}^{n}} \varphi(x, g)+\varphi(x,|\nabla g|) d x \\
& \leqslant \int_{\mathbb{R}^{n}} \varphi(x, g)+\varphi(x,|\nabla f|) d x .
\end{aligned}
$$

Using $0 \leqslant g \leqslant 1,(\mathrm{aInc})_{1},(\mathrm{aDec})_{q}$ and $(\mathrm{A} 0)$, we obtain

$$
\begin{aligned}
\varphi(x, g(x)) & \leqslant \operatorname{Lg}(x) \varphi(x, 1) \leqslant L^{2} g(x) \beta^{-q} \varphi(x, \beta) \\
& \leqslant L^{2} g(x) \beta^{-q} .
\end{aligned}
$$

Integrating over the bounded set $\Omega$ and using the classical Poincaré inequality, we find that

$$
\begin{aligned}
\int_{\Omega} \varphi(x, g) d x & \leqslant C \int_{\Omega} g d x \leqslant C \int_{\Omega} f d x \\
& \leqslant C \int_{\Omega}|\nabla f| d x .
\end{aligned}
$$

Then the embedding $L^{\varphi(\cdot)}(\Omega) \hookrightarrow L^{1}(\Omega)$ (Lemma 5) and Lemma 6 for the function $|\nabla f|$ give that

$$
\int_{\Omega} \varphi(x, g) d x \leqslant C \max \left\{\varrho_{\varphi(\cdot)}(|\nabla f|)^{1 / q}, \varrho_{\varphi(\cdot)}(|\nabla f|)\right\} .
$$

Combining this with (61) and taking the infimum over $f$, we obtain the claim.

In the next proof the Choquet property for Sobolev capacity is needed and hence we assume that $\varphi$ is convex.

Theorem 27. Assume that $\varphi \in \Phi_{c}\left(\mathbb{R}^{n}\right)$ satisfies (A0) and $(\mathrm{aDec})_{q}$. If $\Omega$ is bounded and $E \subset \Omega$, then

$$
C_{\varphi(\cdot)}(E) \leqslant C \max \left\{\operatorname{cap}_{\varphi(\cdot)}(E, \Omega)^{1 / q}, \operatorname{cap}_{\varphi(\cdot)}(E, \Omega)\right\},
$$

where the constant $C$ depends on the dimension, $|\Omega|$, and the constants in (AO) and (aDec).

Proof. We may assume that $\operatorname{cap}_{\varphi(\cdot)}(E, \Omega)<\infty$. By the definition of $\operatorname{cap}_{\varphi(\cdot)}(E, \Omega)$, there exist open sets $U_{i} \supset E$ with $\operatorname{cap}_{\varphi(\cdot)}\left(U_{i}, \Omega\right) \longrightarrow \operatorname{cap}_{\varphi(\cdot)}(E, \Omega)$, as $i \longrightarrow \infty$. Let $U:=\bigcap_{i=1}^{\infty} U_{i}$. Then $U$ is a Borel set, and hence, by the Choquet property (Remark 10),

$$
C_{\varphi(\cdot)}(E) \leqslant C_{\varphi(\cdot)}(U)=\sup _{K \subset U} C_{\varphi(\cdot)}(K),
$$

where the supremum is taken over all compact sets $K \subset U$. By Lemma 26, we obtain

$$
\begin{aligned}
& C_{\varphi(\cdot)}(E) \leqslant \sup _{K} C_{\varphi(\cdot)}(K) \\
& \leqslant C \sup _{K \subset U} \max \left\{\operatorname{cap}_{\varphi(\cdot)}(K, \Omega)^{1 / q}, \operatorname{cap}_{\varphi(\cdot)}(K, \Omega)\right\} \\
& \leqslant C \max \left\{\operatorname{cap}_{\varphi(\cdot)}\left(U_{i}, \Omega\right)^{1 / q}, \operatorname{cap}_{\varphi(\cdot)}\left(U_{i}, \Omega\right)\right\} .
\end{aligned}
$$

The claim follows from this as $i \longrightarrow \infty$. 
From the above result, we can conclude that $C_{\varphi(\cdot)}(E)=0$ if $\operatorname{cap}_{\varphi(\cdot)}(E, \Omega)=0$. To get the converse implication, we first prove the following results.

Lemma 28. Let $\varphi \in \Phi_{w}(\Omega)$ satisfy (aDec). Assume that $C\left(\mathbb{R}^{n}\right) \cap W^{1, \varphi(\cdot)}\left(\mathbb{R}^{n}\right)$ is dense in $W^{1, \varphi(\cdot)}\left(\mathbb{R}^{n}\right)$. If $K \subset \mathbb{R}^{n}$ is compact, then

$$
C_{\varphi(\cdot)}(K)=\inf _{f \in S_{1, \varphi(\cdot)}^{c}(K)} \int_{\mathbb{R}^{n}} \varphi(x, f)+\varphi(x,|\nabla f|) d x,
$$

where $S_{1, \varphi(\cdot)}^{c}(K)=S_{1, \varphi(\cdot)}(K) \cap C\left(\mathbb{R}^{n}\right)$.

Proof. Since $C_{\varphi(\cdot)}(K)$ is defined as the infimum over the larger set $S_{1, \varphi(\cdot)}(K)$, the inequality " $\leqslant$ " is clear.

Suppose $f \in S_{1, \varphi(\cdot)}(K)$, with $0 \leqslant f \leqslant 1$ and choose functions $f_{j} \in C\left(\mathbb{R}^{n}\right) \cap W^{1, \varphi(\cdot)}\left(\mathbb{R}^{n}\right)$ converging to $f$ in $W^{1, \varphi(\cdot)}\left(\mathbb{R}^{n}\right)$, with $0 \leqslant f_{j} \leqslant 1$. Choose a bounded neighborhood $U$ of $K$ such that $f=1$ in $U$. Let $\eta \in C^{1}\left(\mathbb{R}^{n}\right), 0 \leqslant \eta \leqslant 1$ with $\eta=1$ in $\mathbb{R}^{n} \backslash U$ and $\eta=0$ in a neighborhood of $K$.

Let $g_{j}:=1-\left(1-f_{j}\right) \eta$ and note that $g_{j} \geqslant 0$ since $0 \leqslant$ $f_{j}, \eta \leqslant 1$. We find that $f-g_{j}=f-1+\eta-\eta f_{j}=\left(f-f_{j}\right) \eta+$ $(1-\eta)(f-1)=\left(f-f_{j}\right) \eta$, as $f=1$ in $U$ and $\eta=1$ in $\mathbb{R}^{n} \backslash U$. Since $\eta \in W^{1, \infty}\left(\mathbb{R}^{n}\right)$ and $f_{j} \longrightarrow f$ in $W^{1, \varphi(\cdot)}\left(\mathbb{R}^{n}\right)$, we find that $g_{j} \longrightarrow f$ in $W^{1, \varphi(\cdot)}\left(\mathbb{R}^{n}\right)$. Further, $\eta=0$ in a neighborhood of $K$, so $g_{j}=1$ in a neighborhood of $K$. Thus $g_{j} \in S_{1, \varphi(\cdot)}^{c}(K)$. This and $g_{j} \longrightarrow f$ imply the " $\geqslant$ " inequality.

Proposition 29. Let $\Omega \subset \mathbb{R}^{n}$ be bounded and $\varphi \in \Phi_{w}\left(\mathbb{R}^{n}\right)$ satisfy $(a D e c)$. Assume that $C\left(\mathbb{R}^{n}\right)$ is dense in $W^{1, \varphi(\cdot)}\left(\mathbb{R}^{n}\right)$. If $E \subset \Omega$ with $C_{\varphi(\cdot)}(E)=0$, then $\operatorname{cap}_{\varphi(\cdot)}(E, \Omega)=0$.

Proof. Let $K \subset \Omega$ be compact with $C_{\varphi(\cdot)}(K)=0$. By Lemma 28 we may choose a sequence of functions $\left(f_{i}\right)$ from $S_{1, \varphi(\cdot)}^{c}(K)$ such that $\left\|f_{i}\right\|_{W^{1, \varphi(\cdot)}\left(\mathbb{R}^{n}\right)} \longrightarrow 0$. Let $\eta \in C_{0}^{\infty}(\Omega)$ be a cut-off function that equals two in $K$. Since $f_{i} \in S_{1, \varphi(\cdot)}^{c}(K)$, it is easy to conclude that $\eta f_{i}>1$ in $K$ and $\eta f_{i} \geqslant 0$, hence $\eta f_{i} \in R_{\varphi(\cdot)}(K, \Omega)$. Since modular convergence and norm convergence are equivalent [18, Theorem 2.1.11], we obtain

$$
\begin{aligned}
\operatorname{cap}_{\varphi(\cdot)}(K, \Omega) & \leqslant \varrho_{\varphi(\cdot)}\left(\nabla\left(\eta f_{i}\right)\right) \\
& \leqslant \varrho_{\varphi(\cdot)}\left(c f_{i}\right)+\varrho_{\varphi(\cdot)}\left(c\left|\nabla f_{i}\right|\right) \longrightarrow 0 .
\end{aligned}
$$

Hence the claim holds for compact sets.

By (S3) there exists a sequence of open sets $U_{i} \supset E$ with $C_{\varphi(\cdot)}\left(U_{i}\right) \longrightarrow 0$ as $i \longrightarrow \infty$. Let $U:=\bigcap_{i=1}^{\infty} U_{i} \cap \Omega$. Then, $U$ is a Borel set containing $E$ which satisfies $C_{\varphi(\cdot)}(U)=0$. By the Choquet property of the relative capacity, we obtain

$$
\operatorname{cap}_{\varphi(\cdot)}(E, \Omega) \leqslant \operatorname{cap}_{\varphi(\cdot)}(U, \Omega)=\sup _{K} \operatorname{cap}_{\varphi(\cdot)}(K, \Omega),
$$

where the supremum is taken over all compact sets $K \subset U$. By the first part of the proof, $\operatorname{cap}_{\varphi(\cdot)}(K, \Omega)=0$, and the claim follows.

If $E \subset B$ and $\Omega=2 B$ for a ball $B$, then the Lipschitz constant of the cut-off function $\eta$ in the previous proof can be chosen to be $c(\operatorname{diam} B)^{-1}$. Then an similar proof gives the following quantitative version of the previous result, cf. [18, Theorem 10.3.5].

Theorem 30. Let $B \subset \mathbb{R}^{n}$ be a ball and $\varphi \in \Phi_{w}\left(\mathbb{R}^{n}\right)$ satisfy $(a D e c)_{q}$. Assume that $C\left(\mathbb{R}^{n}\right)$ is dense in $W^{1, \varphi(\cdot)}\left(\mathbb{R}^{n}\right)$. For $E \subset B$,

$$
\begin{aligned}
& \operatorname{cap}_{\varphi(\cdot)}(E, 2 B) \\
& \quad \leqslant C\left(1+\max \left\{\operatorname{diam}(B), \operatorname{diam}(B)^{-q}\right\}\right) C_{\varphi(\cdot)}(E)
\end{aligned}
$$

where the constant $C$ depends on $q$ and $L$.

\section{Data Availability}

No data were used to support this study.

\section{Conflicts of Interest}

The authors declare that they have no conflicts of interest.

\section{Acknowledgments}

Debangana Baruah was supported financially by the University of Turku Graduate School MATTI-program.

\section{References}

[1] P. Marcellini, "Regularity of minimizers of integrals of the calculus of variations with nonstandard growth conditions," Archive for Rational Mechanics and Analysis, vol. 105, no. 3, pp. 267-284, 1989.

[2] V. V. Zhikov, "Averaging of functionals of the calculus of variations and elasticity theory," Izvestiya Rossiiskoi Akademii Nauk, vol. 50, no. 4, pp. 675-710, 1986 (Russian).

[3] M. Ružička, Electrorheological Fluids: Modeling and Mathematical Theory, vol. 1748 of Lecture Notes in Mathematics, Springer, Berlin, Germany, 2000.

[4] P. Baroni, M. Colombo, and G. Mingione, "Harnack inequalities for double phase functionals," Nonlinear Analysis, vol. 121, pp. 206-222, 2015.

[5] P. Baroni, M. Colombo, and G. Mingione, "Nonautonomous functionals, borderline cases and related function classes," St. Petersburg Mathematical Journal, vol. 27, no. 3, pp. 347-379, 2015.

[6] P. Baroni, M. Colombo, and G. Mingione, "Regularity for general functionals with double phase," Calculus of Variations and Partial Differential Equations, vol. 57, no. 62, 48 pages, 2018.

[7] M. Colombo and G. Mingione, "Regularity for double phase variational problems," Archive for Rational Mechanics and Analysis, vol. 215, no. 2, pp. 443-496, 2015.

[8] S.-S. Byun and J. Oh, "Global gradient estimates for nonuniformly elliptic equations," Calculus of Variations and Partial Differential Equations, vol. 56, no. 2, article no. 46, 36 pages, 2017.

[9] S.-S. Byun, S. Ryu, and P. Shin, "Calderon-Zygmund estimates for omega-minimizers of double phase variational problems," Applied Mathematics Letters, vol. 86, pp. 256-263, 2018.

[10] A. Clop, R. Giova, and F. Hatami, "Passarelli di, Napoli: Congested traffic dynamics and very degenerate elliptic equations under supercritical Sobolev regularity," Preprint, 2018. 
[11] G. Cupini, F. Giannetti, R. Giova, and A. Passarelli di Napoli, "Regularity results for vectorial minimizers of a class of degenerate convex integrals," Journal of Differential Equations, vol. 265, no. 9, pp. 4375-4416, 2018.

[12] J. Ok, "Regularity of $\omega$-minimizers for a class of functionals with non-standard growth," Calculus of Variations and Partial Differential Equations, vol. 56, no. 2, article no. 48, 31 pages, 2017.

[13] P. Harjulehto, P. Hästö, and O. Toivanen, "Hölder regularity of quasiminimizers under generalized growth conditions," Calculus of Variations and Partial Differential Equations, vol. 56, no. 2, 26 pages, 2017.

[14] P. Gwiazda, I. Skrzypczak, and A. Zatorska-Goldstein, "Existence of renormalized solutions to elliptic equation in MusielakOrlicz space," Journal of Differential Equations, vol. 264, no. 1, pp. 341-377, 2018.

[15] P. Gwiazda, I. Skrzypczak, and A. Zatorska-Goldstein, "Wellposedness of parabolic equations in the non-reflexive and anisotropic Musielak-Orlicz spaces in the class of renormalized solutions," Journal of Differential Equations, vol. 265, no. 11, 2018.

[16] P. Harjulehto, P. Hästö, and R. Klén, "Generalized Orlicz spaces and related PDE," Nonlinear Analysis, vol. 143, pp. 155-173, 2016.

[17] J. Heinonen, T. Kilpelainen, and O. Martio, Nonlinear Potential Theory Of Degenerate Elliptic Equations, Oxford University Press, New York, NY, USA, 1993.

[18] L. Diening, P. Harjulehto, P. Hästö, and M. Ruzicka, Lebesgue and Sobolev Spaces with Variable Exponents, Springer, Berlin, Germany, 2011.

[19] T. Ohno and T. Shimomura, "Musielak-Orlicz-Sobolev spaces with zero boundary values on metric measure spaces," Czechoslovak Mathematical Journal, vol. 66(141), no. 2, pp. 371-394, 2016.

[20] P. Harjulehto and P. Hästö, "Uniform convexity and associate spaces," Czechoslovak Mathematical Journal, pp. 1-10, 2018.

[21] P. A. Hästö, "The maximal operator on generalized Orlicz spaces," Journal of Functional Analysis, vol. 269, no. 12, pp. 40384048, 2015.

[22] G. Choquet, “Theory of capacities," Annales de l'Institut Fourier, vol. 5, pp. 131-295, 1954.

[23] L. C. Evans and R. F. Gariepy, Measure Theory and Fine Properties of Functions, Studies in Advanced Mathematics, CRC Press, Boca Raton, FL, USA, 1992. 


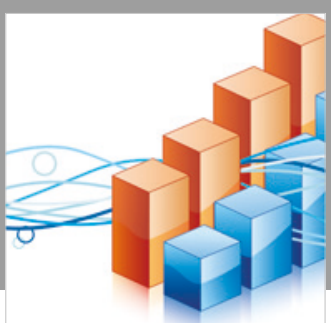

Advances in

Operations Research

\section{-n-m}
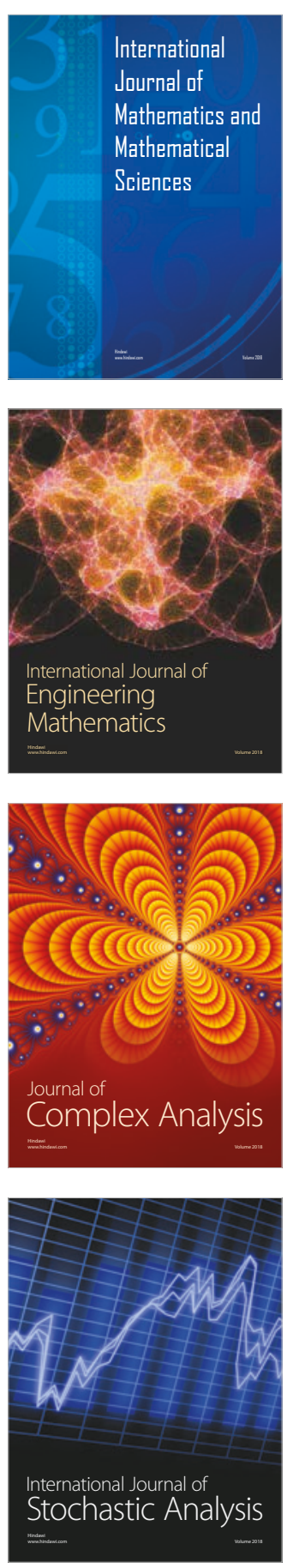
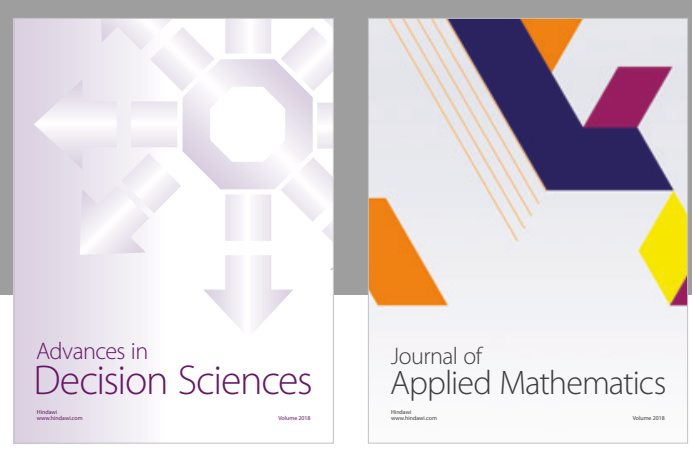

Journal of

Applied Mathematics
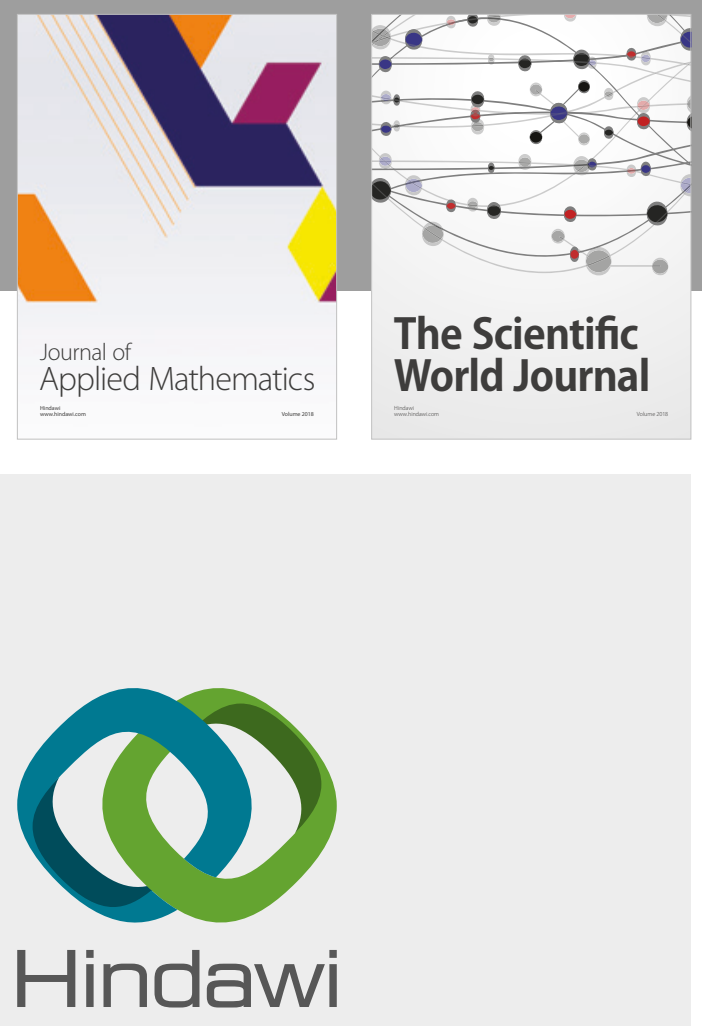

Submit your manuscripts at

www.hindawi.com

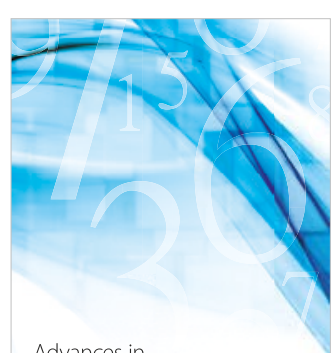

Advances in
Numerical Analysis
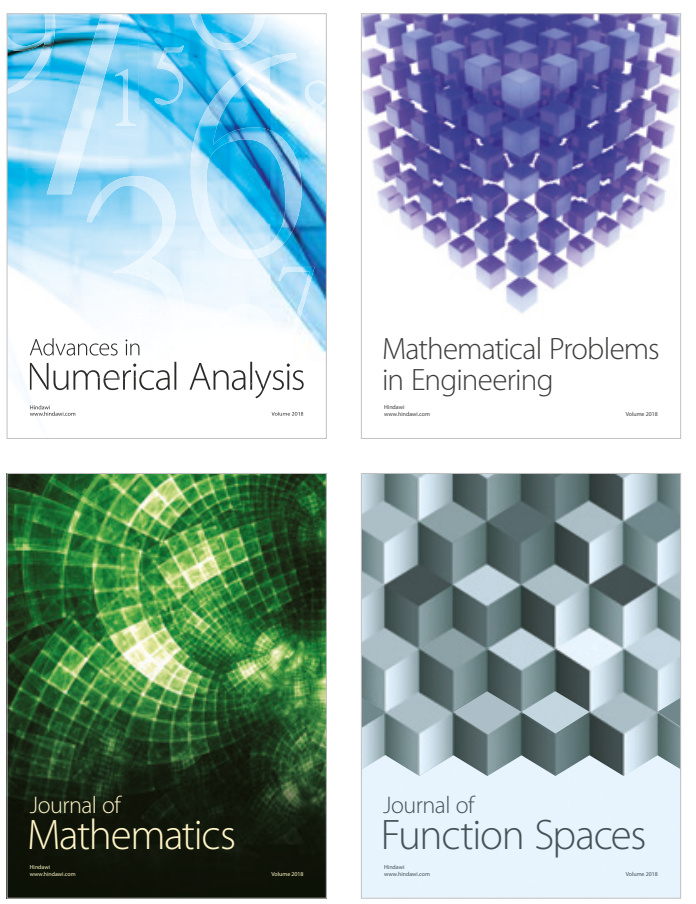

Mathematical Problems in Engineering

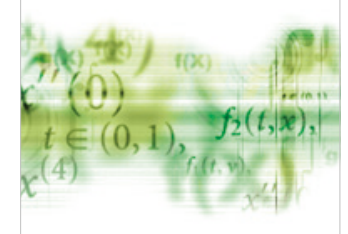

International Journal of

Differential Equations

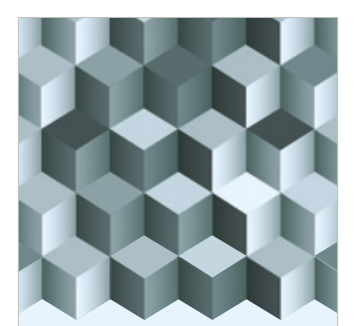

Journal of

Function Spaces

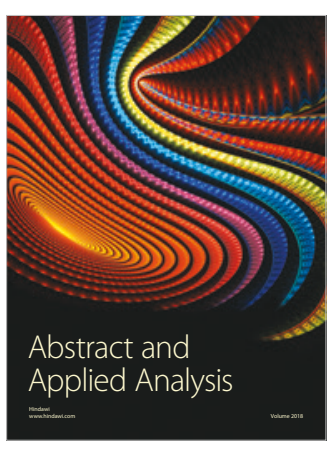

The Scientific

World Journal

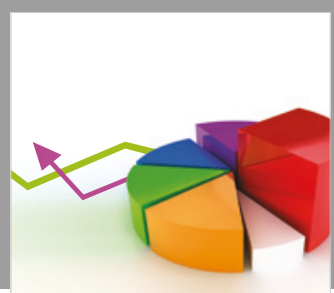

Journal of

Probability and Statistics
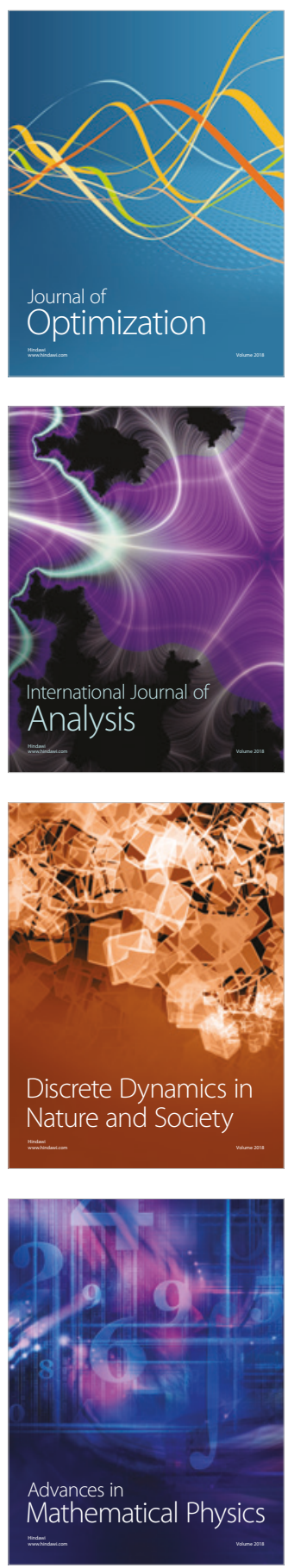